\title{
Transactions on Large-Scale Data- and Knowledge-Centered Systems XXXIII
}

\author{
Abdelkader Hameurlain ${ }^{1}$, Josef Küng ${ }^{2}$, Roland Wagner ${ }^{2}$, Reza Akbarinia ${ }^{3}$, Esther Pacitti ${ }^{3}$ \\ 1 IRIT - Institut de recherche en informatique de Toulouse \\ 2 JKU - Johannes Kepler University Linz [linz] \\ 3 INRIA \& LIRMM, Univ. Montpellier
}

Preface: The LNCS journal Transactions on Large-Scale Data- and Knowledge-Centered Systems focuses on data management, knowledge discovery, and knowledge processing, which are core and hot topics in computer science. Since the 1990s, the Internet has become the main driving force behind application development in all domains. An increase in the demand for resource sharing across different sites connected through networks has led to an evolution of data- and knowledgemanagement systems from centralized systems to decentralized systems enabling large-scale distributed applications providing high scalability. Current decentralized systems still focus on data and knowledge as their main resource. Feasibility of these systems relies basically on P2P (peer-topeer) techniques and the support of agent systems with scaling and decentralized control. Synergy between grids, P2P systems, and agent technologies is the key to data- and knowledge-centered systems in large-scale environments. This 33rd issue of Transactions on Large-Scale Data- and Knowledge-Centered Systems contains five revised selected regular papers. Topics covered include distributed massive data streams, storage systems, scientific workflow scheduling, cost optimization of data flows, and fusion strategies. 